\title{
Forma y topología de la guerra: Espacios de la incertidumbre en Dobrinja, Sarajevo
}

\author{
Inés Aquilué Junyent ${ }^{\mathbf{1}}$, Javier Ruiz Sánchez ${ }^{\mathbf{2}}$ \\ ${ }^{1}$ Departament d'Urbanisme i Ordenació del Territori, Universitat Politècnica de Catalunya. \\ Barcelona, España. ${ }^{2}$ Departamento de Urbanismo y Ordenación del Territorio, Universidad \\ Politécnica de Madrid. Madrid. España \\ E-mail: 'Ines.aquilue@upc.edu, ${ }^{2}$ javier.ruiz@upm.edu,
}

\begin{abstract}
Resumen. El espacio no es solo una definición geométrica, sino también un conjunto topológico de relaciones. Un enfoque no euclidiano puede resultar adecuado para reconocer las relaciones sociales que emergen de los espacios, especialmente en contingencias inciertas y extremas. En relación a la interpretación topológica del espacio, esta investigación apunta a identificar morfologías urbanas, cuya topología se vuelve cada vez más determinante en situaciones de alta incertidumbre. Este enfoque topológico se ha aplicado en un análisis evolutivo de diferentes espacios urbanos bajo asedio, miedo y conflicto armado. En este caso, se examina el papel de Dobrinja un barrio periférico de Sarajevo durante la Guerra de Bosnia [1992-1995].

El análisis topológico del barrio de Dobrinja ha permitido identificar relaciones espaciales que pueden determinar el conflicto y las relaciones de poder establecidas entre las diversas facciones enfrentadas. Este proceso analitico ha demostrado cómo se transformaron los espacios urbanos contemporáneos, públicos y privados, y cómo se produjo una nueva lectura del espacio, basada en un cambio en el paradigma de uso de los espacios y de sus campos relacionales. La concatenación de espacios de Dobrinja se analiza formal y topológicamente durante la Guerra de Bosnia para examinar el papel de la forma urbana durante el conflicto y señalar qué cambios urbanos provocaron. Los resultados de esta investigación determinaron el proceso relacional que definió el vínculo entre forma y sistema social a lo largo del conflicto. En situaciones de alta incertidumbre, el uso y la importancia colectiva del espacio suelen tergiversarse y el análisis del caso así lo demuestra.
\end{abstract}

Palabras clave: Conflicto; topología urbana; complejidad urbana; forma urbana.

\section{Introducción}

Según Haken, la autoorganización emerge cuando sin existir un orden superior que guie las pautas de comportamiento de cada individuo se obtienen resultados coordinados de entendimiento colectivo (Haken, 1977). Esta capacidad de organización puede producirse bajo condiciones extremas en las que el desarrollo y la evolución se conducen a través de procesos autorreferenciales. Estos procesos de autoorganización, dependen en parte del factor azar, y permiten que la ciudad se mantenga en consecutivos estados estables diversos, impredecibles pero selectivos. En el caso de Dobrinja nos fijamos en cuatro escalas de autoorganización en las que el sistema urbano espacial y el sistema urbano social estuvieron absolutamente enlazados.

Dobrinja es un barrio periférico de Sarajevo, cercano al aeropuerto y con escasa conexión al resto de la ciudad (Bublin, 2006; Donia, 2006). Cuenta con más de 36.000 viviendas, y su situación estratégica entre el aeropuerto 
y el resto de Sarajevo, condicionó su papel y supervivencia durante el Sitio de Sarajevo y la Guerra de Bosnia [1992-1995].

\section{Dobrinja y el Sitio de Sarajevo: Autoorganización y lectura inversa del espacio}

La aparición de la línea de fuego en 1992 en Sarajevo condicionó el uso del espacio urbano, perturbando el desarrollo de la vida cotidiana (Aquilué et al., 2014-2015). La organización espacial de la ciudad se vio alterada y totalmente restringida a causa de los patrones de ofensiva y control de las tropas serbobosnias sobre la ciudad. La necesidad de supervivencia hacia una nueva situación conllevó la necesaria adaptabilidad. En Sarajevo y en Dobrinja, la alteración de la función del sistema urbano provocó un cambio en el sistema social, del que emergieron patrones de autoorganización y lectura inversa que a su vez alteraron el espacio.

En Sarajevo, las condiciones eran estremecedoras, el suministro de agua $\mathrm{y}$ de electricidad era intermitente, el acceso a la ciudad estaba cortado por las vías convencionales (carreteras y ferrocarril), el transporte público no funcionaba -a excepción de algún autobús que cruzaba la ciudad desde Alipašino Polje hasta el Hospital Francés(Prstojević et al., 1993), y el suministro de gas era casi inexistente, el acceso a alimentos y agua se producía únicamente en lugares específicos. En el caso de Dobrinja, se situaron diversos puntos de recogida de agua y alimentos en los que se distribuía la ayuda humanitaria. El cambio en el paradigma de subsistencia condujo a una transformación en la vida urbana de la población civil. Las perturbaciones sufridas produjeron variaciones en el uso del espacio tanto privado como público, puesto que la necesaria autoorganización de las funciones cotidianas de supervivencia condujo a una transmutación de su vínculo con el espacio. Estas funciones estuvieron altamente condicionadas por la falta de abastecimiento energético, de comunicación, de alimentos y también por el flujo restringido de personas y vehículos.

La ciudad entendida como un sistema de relaciones materiales, energéticas y sociales requirió adaptarse a la nueva situación y para ello necesitó evolucionar en sus formas de supervivencia. Frente a la ruptura de la estabilidad apareció la necesaria esfera de la adaptabilidad, entendida como la capacidad de respuesta de un sistema frente al incremento de incertidumbre de su entorno.

Grado 1: Alteraciones en los desplazamientos Como consecuencia del establecimiento de la línea de fuego por parte de los serbobosnios, y dado el intenso volumen de bombardeos que la ciudad sufría, fue necesario construir barricadas de protección con automóviles, contenedores y sacos (Barranco et al. (eds.), 2002). En el caso de Dobrinja, los altos edificios de Dobrinja $\mathrm{V}$ situados en primera línea de fuego funcionaban como pantallas protectoras. El acceso más peligroso era la conexión al pie de Mojmilo hacia Alpašino Polje y el punto más crítico dada su alta importancia en términos de conectividad. En los huecos donde no había edificios se colocaron contenedores para «proteger» el espacio de circulación. $\mathrm{La}$ trinchera de Mojmilo fue construida para dar paso a las mercancías que circulaban a través del túnel que cruzaba el aeropuerto hacia la zona libre, de tal modo que el movimiento se realizaba en los espacios menos expuestos. La movilidad a través de las vías principales se vio gravemente afectada, tanto por el difícil uso de los vehículos a motor, como por la exposición frente al ojo francotirador. Eran flujos redefinidos por las alteraciones en el sistema de transporte, pero también en la morfología construida y en el significado del plano horizontal.

La nueva morfología de los flujos a través del espacio público y la conciencia de la existencia de francotiradores condicionaron los recorridos, variando su trayecto habitual. El cambio en las funciones tergiversó el espacio urbano, no sólo modificando las circulaciones sino también la especificidad de sus usos. La importancia del espacio topológico era clave en la lectura inversa del espacio. Las calles y avenidas, zonas de alta conexión, dejaban de ser espacios de adyacencia, y se convirtieron en grandes vacíos. A su vez, el sistema perdía robustez en tanto que menos conexiones eran posibles, se desvanecía así parte de su 


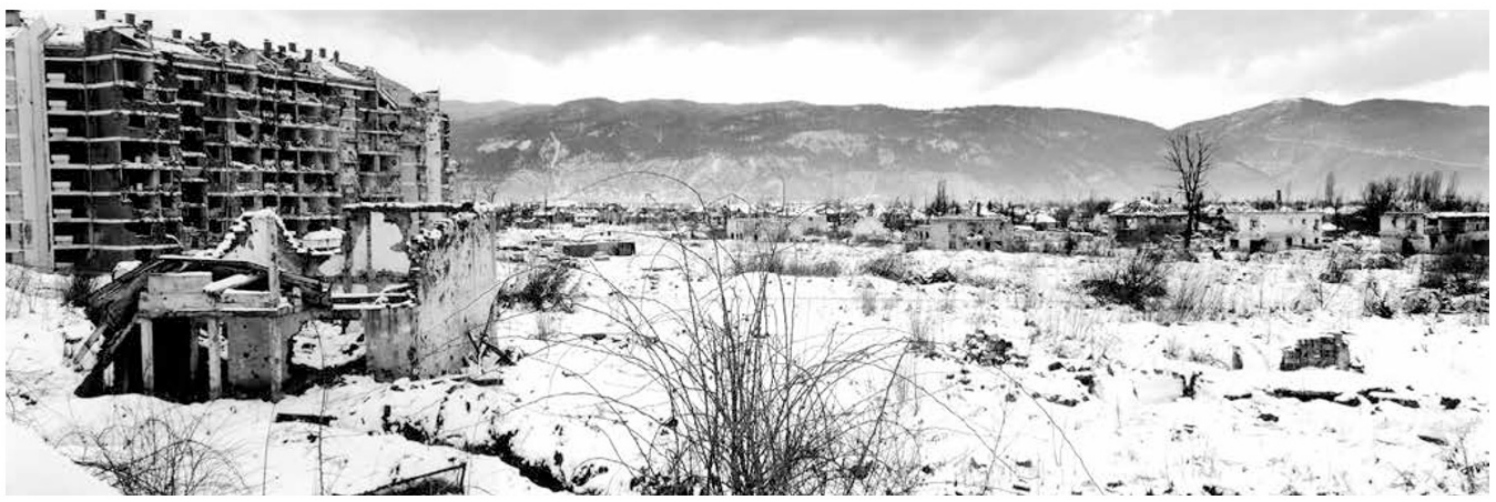

Fig. 1. Primera línea de frente en Dobrinja, 1994. A la izquierda uno de los bloques de Dobrinja V, al fondo las pequeñas edificaciones de C4 y Nedžarići. Fuente: Lowe, 2015, p. 139.

complejidad.

\section{Grado 2: Autoorganización social e institucional: la creación de un hospital y las escuelas-portal}

Dada la difícil comunicación con el resto de la ciudad, en Dobrinja muchas eran las necesidades de los residentes que permanecieron sin ser expulsados o asesinados. Además de la lucha por la supervivencia diaria y por la adquisición de víveres, existía entre los habitantes sitiados de todo Sarajevo una necesaria recuperación de sus rutinas y una vuelta a la normalidad (Jansen, 2014). En Dobrinja, la situación de aislamiento y el fuerte sentido de comunidad ayudaron a constituir un órgano de gobierno popular, a través del que se nominó una Junta de Coordinación que fue aprobada por la Municipalidad de Novi Grad. En la junta diferentes personas se encargaban de la gestión de diversas áreas [servicios de docencia, de salud, económicos, etc.]. Al iniciarse el sitio, se organizó colectivamente un 'centro médico', dada la imposibilidad de desplazar heridos desde Dobrinja hasta los hospitales de la ciudad. Este centro médico de carácter absolutamente improvisado surgió de las necesidades de atención inmediata de los heridos y gracias a los trabajadores de otros centros sanitarios de Sarajevo quienes residían en Dobrinja y no podían desplazarse a diario a sus puestos de trabajo (Karović, 2012).

Miembros de la Junta de Coordinación acordaron fundar el Centro para la docencia de Dobrinja durante la guerra [Ratni nastavni centar Dobrinja] bajo la tutela de Smail Vesnića. En el centro, los trabajadores implantaron un nuevo sistema de escolarización arraigado a la situación física y territorial, dentro del marco de las escuelas de guerra [ratne škole]. Hasta 1992 en Dobrinja existían tres escuelas, sin embargo, ninguno de los tres edificios podía ser utilizado, así que desde el centro se organizó un sistema escolar adecuado a las circunstancias. Se mantenían las tres administraciones de las tres escuelas, aunque no era posible emplear el espacio físico de los tres centros de docencia. Hubo que organizar un nuevo sistema, en el que empleando los tres órganos administrativos centrales, las clases se impartieran en unidades pequeñas esparcidas por los edificios del asentamiento del barrio. Cada unidad se denominó como haustorska škola [escuela-portal], dado que las pequeñas unidades escolares se organizaron en las cajas de escalera, en las partes bajas de los edificios, en los almacenes, en los portales de entrada, en viviendas abandonadas de las plantas bajas y en los cuartos de calderas, siempre en las zonas bajas o semienterradas de los edificios. Pequeños grupos de adultos se organizaban para fundar una unidad escolar, buscaban un lugar más o menos adecuado en los bajos de los edificios y ejercían de profesores sobre grupos reducidos de niños. A finales de 1992 en todo el asentamiento operaban veintiocho unidades escolares con aproximadamente 900 estudiantes y 89 profesores, quienes tenían profesiones muy diversas (Berman, 2005). Empleando esta misma estrategia de subdivisión en unidades, se llegó a fundar un instituto de segundo grado 
previo a la universidad, el Gimnazija Dobrinja, que no había existido hasta el momento, dado que los jóvenes acudían a institutos de otras zonas de la ciudad. Más de 700 alumnos acudieron al instituto organizado a través de múltiples unidades independientes esparcidas por el barrio (Berman, 2005).

Sobre la cuestión espacial, las escuelas dejaron de ser espacios amplios con grandes patios, para convertirse en espacios muy pequeños y estrechos, oscuros y mal iluminados en los que los alumnos debían sentarse en el suelo. Eran espacios de refugio. La elección de este tipo de lugares, y del tamaño reducido de los grupos era una medida de seguridad frente a bombardeos y a francotiradores. Los espacios con menos entidad se convertían en espacios con un significado de arraigo a la comunidad. Las escuelas eran sótanos y almacenes, espacios no habitados antes del sitio. Las unidades escolares de Dobrinja devinieron el modelo de las escuelas de guerra en todo Sarajevo.

Grado 3: Autoorganización en los espacios públicos: lavaderos, cementerios y mercados

Al colapsarse parte de los sistemas comunicativos, materiales y energéticos (alimentos, madera, gas, agua, etc.), el sistema en sus tres niveles debía reorganizarse para no deteriorarse hasta desaparecer. $\mathrm{La}$ autoorganización estaba habitualmente ligada con la supervivencia y una cierta normalización de la vida cotidiana, modificando el uso de los espacios tanto colectivos como privados. Fuera de los estamentos institucionales el día a día requería de la ayuda mutua entre residentes, para la distribución de los pocos recursos. La escasez de alimentos básicos, comida y agua, provocaba la concentración masiva de población civil a la intemperie, especialmente en las líneas de agua, para la recogida de ayuda humanitaria, y también en los mercados improvisados que iban cambiando de localización. Las alteraciones en el significado del espacio condujeron a variaciones en sus funciones también públicas. El pequeño riachuelo de Dobrinja se empleaba como lugar para lavar la ropa (véase figura 2), y los aparcamientos, libres de coches, se convertían de vez en cuando en canchas improvisadas, en las que los adolescentes trataban de escapar de la rutina diaria de la guerra (Karović, 2012). Los puntos de recogida de agua podían ubicarse en propiedades privadas, de manera que espacios de uso habitualmente privado se convertían en espacios colectivos. Los espacios verdes perdieron todos los árboles, utilizados como fuente de energía, y en algunos lugares fueron ocupados por pequeños cementerios. En el centro de Sarajevo, el inmenso cementerio de Koševo debía ser desplazado fuera de los límites urbanos según el Plan Urbanístico de 1986, sin embargo, en vez de ello multiplicó exponencialmente su tamaño.

Los mercados improvisados se abastecían a base del contrabando que aumentó tras la apertura del túnel. Teniendo en cuenta que era posible entrar mercancías de las zonas libres, muchos aprovechaban la situación para poder acarrear bienes a través del túnel entre Butmir y Dobrinja y venderlas en la ciudad a precios muchísimo más elevados (Andreas,

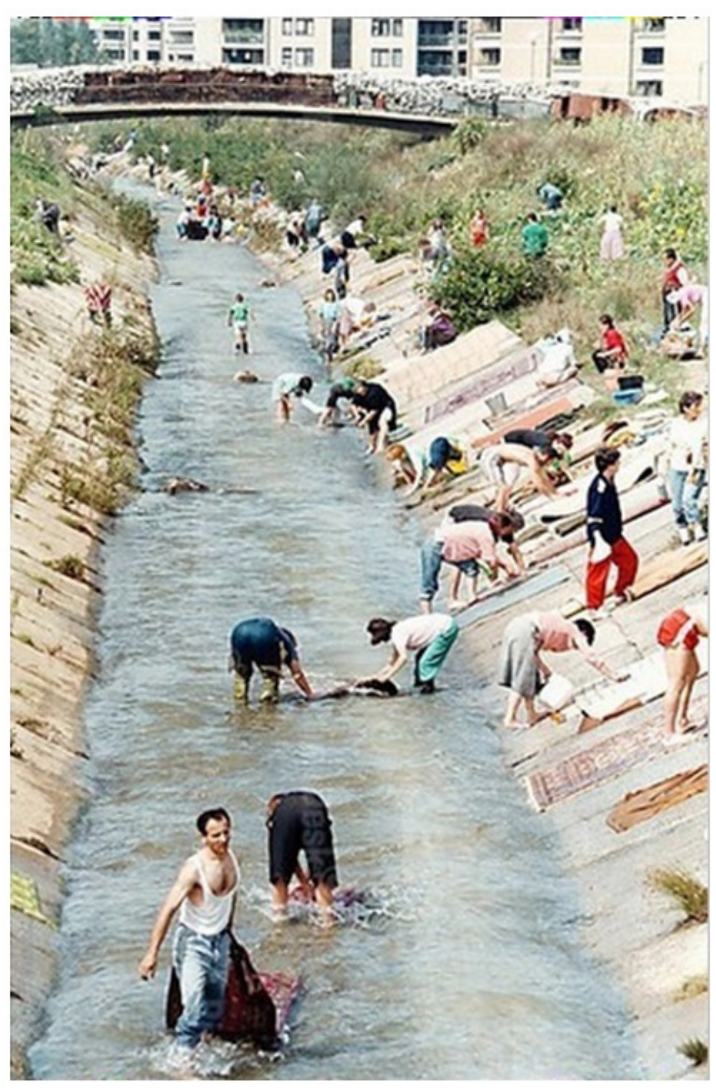

Fig. 2. Arroyo de Dobrinja en el centro del barrio empleado como lavadero, 1995-1992. Fuente: http://forum.klix.ba/dobrinja-1992-1995-ratnadesavanja-p9874782.html 


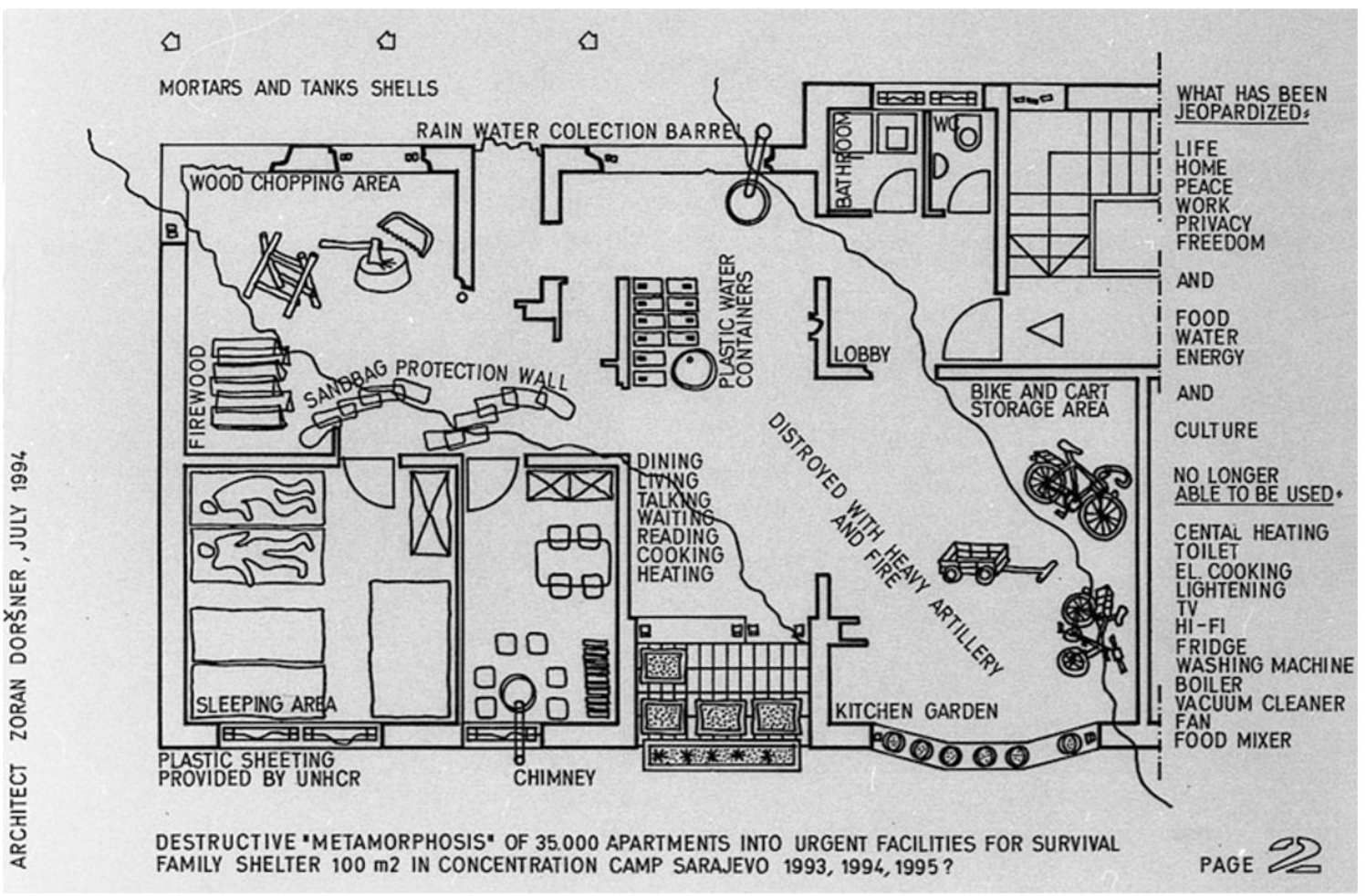

Fig. 3. Esquema realizado por el arquitecto Zoran Doršner sobre los apartamentos del área olímpica convertido en refugio de guerra. Fuente: colección del Museo de Historia de Bosnia-Herzegovina [Historijski Musej Bosne i Hercegovine].

2008). Este tipo de economía provocaba que la compraventa se produjera de manera dispersa y los mercados fueran implementados en lugares diversos. Los habitantes de Dobrinja eran plenamente conscientes del funcionamiento del mercado de estraperlo, puesto que el barrio era la entrada de las mercancías a la ciudad. El camino era claro, desde el túnel hacia Sarajevo por Dobrinja.

El entorno sufrió modificaciones a gran escala y la vida urbana bajo asedio enfatizó las redes entre la población civil y el medio circundante para obtener aquello que requería para abastecerse. Gracias a la autoorganización del espacio que empeñó nuevas funciones y a la autorreferencia del sistema social fue posible la supervivencia del sistema urbano en su conjunto.

\section{Grado 4: Alteraciones en la vivienda}

La reorganización de la vida cotidiana también afectó el espacio más privado, la vivienda. En primer lugar la ocupación de algunas partes de Dobrinja, así como la destrucción total de los edificios de viviendas situados en la primera línea de fuego, provocaron un cierto éxodo hacia el interior del barrio, en la zona Dobrinja II y III. Algunos habitantes trasladaron su residencia hacia viviendas vacías, abandonadas al inicio de la guerra, a tan sólo unos cientos de metros de su propia vivienda destruida.

La función de los espacios en los edificios de vivienda se alteró. El salón y los pasillos se convirtieron en almacenes de leña, y las estancias perdieron sus funciones específicas. Cada habitante de un bloque era plenamente consciente de que en cualquier momento, bajo el silbido o el estruendo de un proyectil o una granada, debería correr hacia la caja de escalera, juntamente con todos aquellos que residían con él. Cada uno de los individuos que habitaba en un bloque, tenía su lugar en el basamento del edificio donde se refugiaban y agrupaban cuando, tras alcanzar la caja de escalera, buscaban protección frente a la artillería que les amenazaba (Prstojević et al., 1993). También el flujo de personas dentro de la ciudad se realizaba mayormente en las zonas bajas de las construcciones. Las partes emergentes eran 
objetivos de fácil alcance para francotiradores, bombas de mortero y proyectiles, consecuencia de la localización de las tropas serbobosnias en las colinas circundantes. El interior de la vivienda modificaba su distribución, y un nuevo orden extremadamente precario reorganizaba el espacio íntimo (véase figura 3).

\section{Coda}

La falta de recursos y la incapacidad de la administración para gestionar unos abastecimientos cortocircuitados indujeron a que el plano de organización y decisión se mantuviera entre los elementos del sistema no jerarquizado y en el vínculo con el medio más directo. La autoorganización social y espacial aumentó el número de estados accesibles que habían desaparecido tras el colapso de las infraestructuras, deviniendo también un paradigma de la gestión del espacio. En términos de complejidad, la adaptabilidad del sistema condujo a un aumento de la complejidad a partir de una situación de abastecimiento precario. $\mathrm{La}$ creación de nuevas redes de autoorganización supuso un factor de adaptación que dio lugar
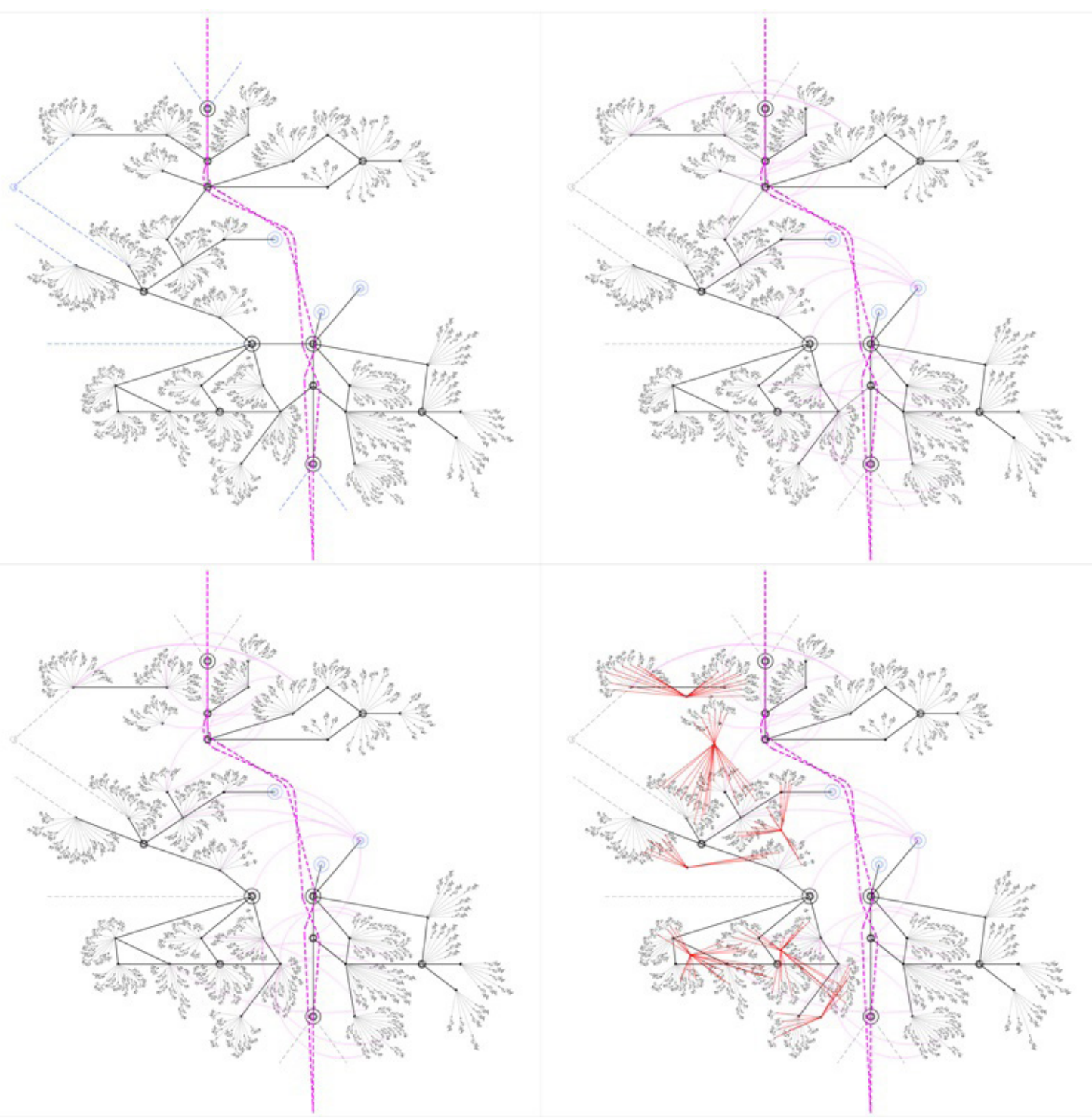

Fig. 4. Representación topológica del ámbito afectado por la línea de fuego en Dobrinja. El análisis topológico del espacio permite analizar el uso por parte del sistema social de las relaciones espaciales.

Fuente: Aquilué, 2017, p. 292. 
a nuevos estados accesibles, puesto que gran parte de los anteriores habían sido destruidos. Para supervivir, además, el sistema social se anticipaba a la incertidumbre, gracias a los cambios en las estrategias de circulación y uso del espacio. Los espacios abiertos variaron su significado, ya que el sistema tuvo que anticiparse a las agresiones del entorno.

La lectura inversa del espacio se produce cuando existe un cambio en su cognición, en tanto que su legibilidad se ve absolutamente modificada a causa de la incertidumbre (Weizman, 2007; Portugali 2011). En Sarajevo, donde se localizaban nodos y arterias de comunicación, durante el sitio los supervivientes identificaban espacios de muerte y exposición; donde se hallaba la intimidad de la vivienda, aparecían multitudes en busca de agua; y donde los vecinos almacenaban trastos, se educaron a los niños de Dobrinja.

La figura 4 identifica topológicamente estas modificaciones relacionales acaecidas en Dobrinja. La aparición de la línea de fuego (en magenta), y el asedio producido desde las líneas de fuego serbobosnias provocó la desconexión entre ambos lados, y la aparición de nuevos campos relacionales (en rojo), alejados del uso del espacio público. El espacio en Dobrinja no sólo sufrió agresiones materiales, sino que se produjo una tergiversación de su lectura funcional y relacional.

\section{Referencias}

Andreas, Peter. 2008. Blue Helmets and black markets: The business of survival in the Siege of Sarajevo. Nueva York: Cornell University Press.

Aquilué, Inés; Leković, Milica; Ruiz, Javier. 2014-2015. "Urban Trauma and Selforganization of the City. Autopoiesis in the Battle of Mogadishu and the Siege of Sarajevo". Urban 8-9: 63-76.

Aquilué, Inés. 2017. El urbanismo de la seguridad frente a la complejidad urbana. Morfologías contemporáneas del miedo y el asedio. Bijlmermeer [Ámsterdam], Dobrinja [Sarajevo] y el Distrito Central de Beirut. Tesis doctoral dirigida por Estanislao Roca Blanch y Javier Ruiz Sánchez. Barcelona:
Departament d'Urbanisme i Ordenació del Territori, Universitat Politècnica de Catalunya.

Barranco, Alonso; Poncelas, Milde; Hauck, Eric; Riu, Anna (eds.). 2002. Živjelo Sarajevo! La ciutat en la nostra memòria. Barcelona: Ajuntament de Barcelona.

Berman, David M. 2005. "The war schools of Dobrinja: Schooling under siege in a Sarajevo community". The Carl Beck Papers in Russian \& East European Studies 1705: 1-64.

Bublin, Mehmed. 2006. Sarajevo u istoriji: od neolitskog naselja do metropolisa. Sarajevo: Buybook.

Donia, Robert J. 2006. Sarajevo a Biography. Londres: Hurst \& Company.

Haken, Hermann. 1977. Synergetics: An introduction. Nonequilibrium phase transitions and self-organization in physics, chemistry, and biology. Berlín, Heidelberg y Nueva York: Springer.

Jansen, Stef. 2014. "Hope for/against the State: Gridding in a besieged Sarajevo suburb". Ethnos 79(2): 238-260.

Karović, Merisa. 2012. "Zločini nad civilima u sarajevskom naselju Dobrinja”. Historijska Traganja / Historical Searches 9: 105-158.

Lowe, Paul. 2015. "The Siege of Sarajevo". Photography and Culture 8(1): 135-142.

Prstojević, Miroslav; Puljić, Zeljk; Wagner, Aleksandra; Razović, Maja. 1993. Sarajevo: Survival Guide. Zagreb: FAMA.

Portugali, Juval. 2011. Complexity, Cognition and the City. Berlín, Heidelberg y Nueva York: Springer.

Weizman, Eyal. 2007. Hollow Land: Israel's Architecture of Occupation. Londres y Nueva York: Verso. 